J. Korean Math. Soc. 49 (2012), No. 6, pp. 1163-1174

http://dx.doi.org/10.4134/JKMS.2012.49.6.1163

\title{
SPATIAL DECAY BOUNDS FOR A TEMPERATURE DEPENDENT STOKES FLOW
}

\author{
Jong Chul Song \\ To the memory of my esteemed professor, teacher and friend, Lawrence E. Payne
}

\begin{abstract}
This paper examines a temperature dependent Stokes flow in a semi-infinite cylinder. Under appropriate initial and boundary conditions the author establishes exponential decay of solutions in energy norm with distance from the finite end of the cylinder.
\end{abstract}

\section{Introduction}

We consider a problem of temperature dependent Stokes flow in a semiinfinite cylinder of uniform cross section. With prescribed data on the finite end of the cylinder together with appropriate homogeneous initial conditions and boundary conditions on the lateral surface, Saint-Venant type decay results are established. Other decay results for Darcy flow, Stokes and Navier-Stokes flow have been obtained by Payne and Song [12], Ames et al. [1], Song [14], Lin and Payne [8], and Horgan and Wheeler [6]. See for instance the survey papers of Horgan and Knowles [5], Horgan [3, 4] and the book of Straughan [15].

In describing the geometry of the semi-infinite cylinder we let $R$ denote its interior and $\partial R$ its boundary. The generators of the cylinder are assumed to be parallel to the $x_{3}$ axis whose entry section is assumed to lie in the plane $x_{3}=0$. Denoting the cross section of the pipe by $D$, the closure of $D$ by $\bar{D}$ and its boundary by $\partial D$, we introduce the notation:

$$
\begin{aligned}
& R_{z}=\left\{\left(x_{1}, x_{2}, x_{3}\right) \mid\left(x_{1}, x_{2}\right) \in D, x_{3}>z \geq 0\right\}, \\
& D_{z}=\left\{\left(x_{1}, x_{2}, x_{3}\right) \mid\left(x_{1}, x_{2}\right) \in D, x_{3}=z\right\} .
\end{aligned}
$$

Clearly $R_{0}=R$.

Received February 12, 2011; Revised February 2, 2012.

2010 Mathematics Subject Classification. 35Q30, 35B40, 76 D07.

Key words and phrases. spatial decay bounds, differential inequality, a temperature dependent Stokes flow.

This research was supported by Basic Science Research Program through the National Research Foundation of Korea funded by the Ministry of Education, Science and Technology (NRF 2010-0012215). 
Let $u_{i}(i=1,2,3),$,$p , and T$, all of functions of $\left(x_{1}, x_{2}, x_{3}, t\right)$ denote respectively the velocity field, the pressure, the temperature. The flow is described by

$$
\begin{aligned}
u_{i, t} & =-p_{, i}+\nu \Delta u_{i}+g_{i}(x) T \quad \text { in } R \times\{t>0\}, \\
u_{j, j} & =0 \quad \text { in } R \times\{t>0\}, \\
T_{, t}+u_{i} T_{, i} & =\kappa \Delta T \quad \text { in } R \times\{t>0\}, \\
u_{i}(x, 0) & =0, T(x, 0)=0 \quad \text { in } R \times\{t=0\}, \\
u_{i} & =0, T=0 \quad \text { on } \partial R \backslash D_{0} \times\{t \geq 0\}, \\
u_{i} & =f_{i}\left(x_{1}, x_{2}, t\right), T=F\left(x_{1}, x_{2}, t\right) \quad \text { on } D_{0} \times\{t \geq 0\},
\end{aligned}
$$

where $\Delta$ is the Laplace operator, $\nu$ and $\kappa$ the constant kinematic viscosity and the constant conductivity respectively, $g_{i}$ a given vector function, and a comma is used throughout to denote partial differentiation. We also use the summation convention of summing over Latin subscripts ranging from 1 to 3 and over the Greek subscript $\alpha$ from 1 to 2 unless noted otherwise. The prescribed functions $f_{i}$ and $F$ are assumed to be continuously differentiable in $R$ and to vanish on $\partial D$ for nonnegative $x_{3}$ and $t$, which is to satisfy the compatibility relationships (1.5). For compatibility we further assume that $f_{i, i}=0$ and that $f_{\alpha, \alpha}$ is differentiable. By re-scaling the space and time variables, we may take both $\nu$ and $\kappa$ to be 1 .

We assume that time lies in some finite interval $[0, T]$. We further assume if the data $f_{i}$ and $F$ are sufficiently small in $L_{2}$, a classical solution of the initial-boundary value problem (1.1)-(1.6) will exist.

We note from (1.2) and (1.5) that

$$
\begin{aligned}
\int_{D_{z}} u_{3} d A & =\int_{D_{0}} u_{3} d A+\int_{0}^{z} \int_{D_{\xi}} u_{3,3} d A d \xi \\
& =\int_{D_{0}} u_{3} d A-\int_{0}^{z} \int_{D_{\xi}} u_{\alpha, \alpha} d A d \xi \\
& =\int_{D_{0}} f_{3} d A,
\end{aligned}
$$

where $d A$ denotes the element of area in $D$. If the mean value of $f_{3}$ over $D$ is zero, we expect the solution in some appropriate measure to vanish exponentially (see [1, p. 1399]). However, here we assume that the net entry flow in the pipe

$$
\int_{D} f_{3} d A=Q(t)
$$

is nonzero and we suppose that $T \rightarrow o\left(x_{3}^{-1}\right)$ uniformly in $x, x_{2}, t$ as $x_{3} \rightarrow \infty$ in view of the assumption (1.18), we expect that for sufficiently small data in (1.1)-(1.6) the velocity field $\left(u_{1}, u_{2}, u_{3}\right)$ will tend to a transient Poiseuille flow 
$(0,0, V)$ as $x_{3} \rightarrow \infty$, where $V\left(x_{1}, x_{2}, t\right)$ satisfies

$$
\begin{aligned}
V_{, t} & =V_{, \alpha \alpha}-\hat{p}_{, 3} \quad \text { in } D \times\{t>0\}, \\
V & =0 \quad \text { on } \partial D \times\{t \geq 0\}, \\
V & =0 \quad \text { in } D \times\{t=0\} .
\end{aligned}
$$

The gradient of the pressure $\hat{p}$ in (1.7) has the form $\hat{p}_{, i}=-P \delta_{3 i}$ where $P$ is a positive function of $t$ only. This function $P(t)$ is not prescribed but is determined by the net inflow condition

$$
\int_{D} V\left(x_{1}, x_{2}, t\right) d A=\int_{D} f_{3} d A=Q(t) .
$$

For given $Q(t)$, the problem (1.7)-(1.10) is viewed as an inverse problem for determining $P(t)$ and $V\left(x_{1}, x_{2}, t\right)$ (see [14, pp. 506-507] and [8, pp. 459-460] and refer also to the similar argument for the stationary Navier-Stokes entry flow $[1$, p. 791] and $[6$, p. 99]).

We now let

$$
w_{i}=u_{i}-v_{i}, \quad q_{, i}=p_{, i}-P(t) \delta_{i 3},
$$

where $\left(v_{1}, v_{2}, v_{3}\right)=(0,0, V)$. Then we rewrite $(1.1)-(1.6)$ as

$$
\begin{aligned}
w_{i, t} & =-q_{, i}+\Delta w_{i}+g_{i}(x) T \quad \text { in } R \times\{t>0\}, \\
w_{j, j} & =0 \quad \text { in } R \times\{t>0\}, \\
T_{, t}+\left(w_{i}+v_{i}\right) T_{, i} & =\Delta T \quad \text { in } R \times\{t>0\}, \\
w_{i}(x, 0) & =0, T(x, 0)=0 \quad \text { in } R \times\{t=0\}, \\
w_{i} & =0, T=0 \quad \text { on } \partial R \backslash D_{0} \times\{t \geq 0\}, \\
w_{i} & =f_{i}-V \delta_{i 3}, T=F\left(x_{1}, x_{2}, t\right) \quad \text { on } D_{0} \times\{t \geq 0\} .
\end{aligned}
$$

We assume further that for any finite positive constants $k_{1}$ and $k_{2}$ the weighted energy expression

$$
\int_{0}^{t} \int_{R} x_{3} w_{i, j} w_{i, j} d x d \eta+k_{1} \int_{0}^{t} \int_{R} x_{3} w_{i, \eta} w_{i, \eta} d x d \eta+k_{3} \int_{0}^{t} \int_{R} x_{3} T_{, i} T_{, i} d x d \eta
$$

is bounded. Here $d x$ denotes the element of volume and $d \eta$ the element of time and there is no summation over a running time variable $\eta$.

In the next section we record some of the inequalities that will be used in our derivations. In Section 3 we derive a differential inequality for a weighted energy which integrates to yield exponential decay for finite energy solutions, and in the final section we establish a bound for the weighted total energy. To attempt to derive the absolute sharpest result would lead to a long and perhaps confusing paper, so in this paper we do not attempt to determine optimal results. Some detail, however, is necessary in order to show that the bounds we obtain are actually valid. 


\section{Auxiliary inequalities}

We list in this section a number of inequalities in addition to the Schwarz inequality and the arithmetic-geometric mean inequality used throughout this paper.

Let $v$ be a Dirichlet integrable function defined on a bounded plane domain $D$ and vanishing on $\partial D$, then

$$
\lambda \int_{D} v^{2} d A \leq \int_{D} v_{, \alpha} v_{, \alpha} d A
$$

where $\lambda$ is the smallest eigenvalue of

$$
w, \alpha \alpha+\hat{\lambda} w=0 \quad \text { in } D, \quad w=0 \quad \text { on } \partial D .
$$

Lower bounds for $\lambda$ are well known (see [9]).

We also make use of the following lemma.

Lemma. Let $R$ be a bounded simply connected region in $\mathbb{R}^{3}$ with Lipschitz boundary $\partial R$. Then, given any a Dirichlet integrable function $v$ satisfying $\int_{R} v d x=0$, there exists a vector field with components $\chi_{i}(i=1,2,3)$ which is Dirichlet integrable and vanishes on $\partial R$ and a dimensionless constant $C$ depending only on the geometry of $R$ such that

$$
\chi_{j, j}=v \quad \text { in } R
$$

and

$$
\int_{R} \chi_{i, j} \chi_{i, j} d x \leq C \int_{R}\left[\chi_{j, j}\right]^{2} d x
$$

This lemma is established by Ladyzhenskaya and Solonnikov [7] and in two dimensions this inequality by Babusuka and Aziz [2] (see also Horgan and Wheeler [6]). Recently, a bound for the optimal constant $C$ is obtained by Payne [10].

\section{Decay bounds}

We now consider the energy expression for any finite positive constants $k_{1}$ and $k_{2}$,

$$
\begin{aligned}
F(z, t)= & \int_{0}^{t} \int_{R_{z}}(\xi-z) w_{i, j} w_{i, j} d x d \eta+k_{1} \int_{0}^{t} \int_{R_{z}}(\xi-z) w_{i, \eta} w_{i, \eta} d x d \eta \\
& +k_{2} \int_{0}^{t} \int_{R_{z}}(\xi-z) T_{, i} T_{, i} d x d \eta \\
= & I_{1}+I_{2}+I_{3},
\end{aligned}
$$

where positive parameters $k_{1}$ and $k_{2}$ are to be determined later. By assumption (1.18), $F(z, t)$ is clearly bounded. Our goal is to show that for specific choices 
of $k_{1}$ and $k_{2}, F(z, t)$ actually decays exponentially in $z$. Upon integration by parts and using (1.12)-(1.16), we obtain

$$
\begin{aligned}
I_{1}= & -\int_{0}^{t} \int_{R_{z}} w_{i} w_{i, 3} d x d \eta+\int_{0}^{t} \int_{R_{z}} w_{3} q d x d \eta+\int_{0}^{t} \int_{R_{z}}(\xi-z) w_{i} g_{i} T d x d \eta \\
& -\left.\frac{1}{2} \int_{R_{z}}(\xi-z) w_{i} w_{i} d x\right|_{\eta=t},
\end{aligned}
$$

$$
\begin{aligned}
I_{2}= & k_{1} \int_{0}^{t} \int_{R_{z}} w_{3, \eta} q d x d \eta-k_{1} \int_{0}^{t} \int_{R_{z}} w_{i, \eta} w_{i, 3} d x d \eta \\
& +k_{1} \int_{0}^{t} \int_{R_{z}}(\xi-z) w_{i, \eta} g_{i} T d x d \eta-\left.\frac{k_{1}}{2} \int_{R_{z}}(\xi-z) w_{i, j} w_{i, j} d x\right|_{\eta=t},
\end{aligned}
$$

$$
I_{3}=-k_{2} \int_{0}^{t} \int_{R_{z}} T T_{, 3} d x d \eta+\frac{k_{2}}{2} \int_{0}^{t} \int_{R_{z}}\left(w_{3}+v_{3}\right) T^{2} d x d \eta-\frac{k_{2}}{2} \int_{R_{z}} T^{2} d x .
$$

In bounding $I_{3}$, we assume that the velocity is uniformly bounded in $R$. This allows us to conclude that $T$ satisfies a maximum principle in $R$. Integrating by parts, applying Schwarz's inequality, the arithmetic-geometric mean inequality, and (2.1) in (3.2)-(3.4), and dropping negative terms, we have

$$
\begin{aligned}
I_{1} \leq & \left(\frac{1}{\lambda} \int_{0}^{t} \int_{R_{z}} w_{i, \alpha} w_{i, \alpha} d x d \eta\right)^{1 / 2}\left(\int_{0}^{t} \int_{R_{z}} w_{i, 3} w_{i, 3} d x d \eta\right)^{1 / 2} \\
& +\int_{0}^{t} \int_{R_{z}} w_{3} q d x d \eta \\
& +\frac{g}{\lambda}\left(\int_{0}^{t} \int_{R_{z}}(\xi-z) w_{i, j} w_{i, j} d x d \eta\right)^{1 / 2}\left(\int_{0}^{t} \int_{R_{z}}(\xi-z) T_{, i} T_{, i} d x d \eta\right)^{1 / 2}, \\
I_{2} \leq & k_{1} \int_{0}^{t} \int_{R_{z}} w_{3, \eta} q d x d \eta-k_{1} \int_{0}^{t} \int_{R_{z}} w_{i, \eta} w_{i, 3} d x d \eta \\
& +k_{1}\left(\int_{0}^{t} \int_{R_{z}}(\xi-z) w_{i, \eta} w_{i, \eta} d x d \eta\right)^{1 / 2}\left(\frac{g^{2}}{\lambda} \int_{0}^{t} \int_{R_{z}}(\xi-z) T_{, i} T_{, i} d x d \eta\right)^{1 / 2}, \\
I_{3} \leq & k_{2}\left(\frac{1}{\lambda} \int_{0}^{t} \int_{R_{z}} T_{, \alpha} T_{, \alpha} d x d \eta\right)^{1 / 2}\left(\int_{0}^{t} \int_{R_{z}} T_{, 3} T_{, 3} d x d \eta\right)^{1 / 2} \\
& +\frac{k_{2} T_{M}}{2}\left(\int_{0}^{t} \int_{R_{z}} w_{3}^{2} d x d \eta\right)^{1 / 2}\left(\frac{1}{\lambda} \int_{0}^{t} \int_{R_{z}} T_{, \alpha} T_{, \alpha} d x d \eta\right)^{1 / 2} \\
& +\frac{k_{2}|V|_{M}}{2 \lambda} \int_{0}^{t} \int_{R_{z}} T_{, \alpha} T_{, \alpha} d x d \eta,
\end{aligned}
$$


where

$$
g=\max _{\bar{D}}\left(g_{i} g_{i}\right)^{1 / 2}, \quad T_{M}=\max _{D \times\{t>0\}} F\left(x_{1}, x_{2}, t\right), \quad|V|_{M}=|V|_{\max },
$$

with $|V|_{M}$ given in [8]. Substituting the bounds for $I_{1}, I_{2}$, and $I_{3}$ into (3.1), we have

$$
\begin{aligned}
& \left(1-\frac{\epsilon_{1}}{2 \lambda}\right) \int_{0}^{t} \int_{R_{z}}(\xi-z) w_{i, j} w_{i, j} d x d \eta \\
& +k_{1}\left(1-\frac{\epsilon_{2}}{2 \lambda}\right) \int_{0}^{t} \int_{R_{z}}(\xi-z) w_{i, \eta} w_{i, \eta} d x d \eta \\
& +\left(k_{2}-\frac{g^{2}}{2 \epsilon_{1} \lambda}-\frac{k_{1} g^{2}}{2 \epsilon_{2} \lambda}\right) \int_{0}^{t} \int_{R_{z}}(\xi-z) T_{, i} T_{, i} d x d \eta \\
(3.5) \leq & \frac{1}{\sqrt{\lambda}} \int_{0}^{t} \int_{R_{z}} w_{i, j} w_{i, j} d x d \eta+\int_{0}^{t} \int_{R_{z}} w_{3} q d x d \eta+k_{1} \int_{0}^{t} \int_{R_{z}} w_{3, \eta} q d x d \eta \\
& -k_{1} \int_{0}^{t} \int_{R_{z}} w_{i, \eta} w_{i, 3} d x d \eta+\frac{k_{2}}{2 \sqrt{\lambda}} \int_{0}^{t} \int_{R_{z}} T_{, i} T_{, i} d x d \eta \\
& +\frac{k_{2} T_{M}}{2}\left(\int_{0}^{t} \int_{R_{z}} w_{3}^{2} d x d \eta\right)^{1 / 2}\left(\frac{1}{\lambda} \int_{0}^{t} \int_{R_{z}} T_{, \alpha} T_{, \alpha} d x d \eta\right)^{1 / 2} \\
& +\frac{k_{2}|V|_{M}}{2 \lambda} \int_{0}^{t} \int_{R_{z}} T_{, \alpha} T, \alpha d x d \eta
\end{aligned}
$$

for positive constants $k_{1}$ and $k_{2}$ to be specified. Choosing

$$
k_{1}=\frac{1}{\lambda}, k_{2}=\frac{1}{2}+\frac{g^{2}}{\lambda^{2}}, \epsilon_{1}=\lambda, \epsilon_{2}=1
$$

we have

$$
\begin{aligned}
& \frac{1}{2} \int_{0}^{t} \int_{R_{z}}(\xi-z) w_{i, j} w_{i, j} d x d \eta+\frac{1}{2 \lambda} \int_{0}^{t} \int_{R_{z}}(\xi-z) w_{i, \eta} w_{i, \eta} d x d \eta \\
& +\frac{1}{2} \int_{0}^{t} \int_{R_{z}}(\xi-z) T_{, i} T_{, i} d x d \eta \\
& \leq \frac{1}{\sqrt{\lambda}} \int_{0}^{t} \int_{R_{z}} w_{i, j} w_{i, j} d x d \eta+\int_{0}^{t} \int_{R_{z}} u_{3} q d x d \eta+k_{1} \int_{0}^{t} \int_{R_{z}} w_{3, \eta} q d x d \eta \\
& \quad-k_{1} \int_{0}^{t} \int_{R_{z}} w_{i, \eta} w_{i, 3} d x d \eta+\frac{k_{2}}{2 \sqrt{\lambda}} \int_{0}^{t} \int_{R_{z}} T_{, i} T_{, i} d x d \eta \\
& \quad+\frac{k_{2} T_{M}}{2}\left(\int_{0}^{t} \int_{R_{z}} w_{3}^{2} d x d \eta\right)^{1 / 2}\left(\frac{1}{\lambda} \int_{0}^{t} \int_{R_{z}} T_{, \alpha} T_{, \alpha} d x d \eta\right)^{1 / 2} \\
& \quad+\frac{k_{2}|V|_{M}}{2 \lambda} \int_{0}^{t} \int_{R_{z}} T_{, \alpha} T_{, \alpha} d x d \eta
\end{aligned}
$$


We now set

$$
\begin{aligned}
E(z, t)= & \frac{1}{2} \int_{0}^{t} \int_{R_{z}}(\xi-z) w_{i, j} w_{i, j} d x d \eta+\frac{1}{2 \lambda} \int_{0}^{t} \int_{R_{z}}(\xi-z) w_{i, \eta} w_{i, \eta} d x d \eta \\
& +\frac{1}{2} \int_{0}^{t} \int_{R_{z}}(\xi-z) T_{, i} T_{, i} d x d \eta
\end{aligned}
$$

from which we seek to derive a first-order differential inequality. We, therefore, bound the terms on the right in (3.7) in terms of $-\frac{\partial E}{\partial z}$. Upon Schwarz's inequality and the arithmetic-geometric mean inequality, we can easily estimate most of terms except for the two terms involving the pressure. To seek a bound for $\int_{0}^{t} \int_{R_{z}} w_{3} q d x d \eta$, we note that, for any $z \geq 0$,

$$
\int_{R_{z}} w_{3} d x=0
$$

Accordingly by Lemma, there exists a vector function $\chi_{i}$ such that

$$
\chi_{i, i}=w_{3} \quad \text { in } R_{z}, \quad \chi_{i}=0 \quad \text { on } \partial R_{z}
$$

and for $\chi_{i}$ inequality (2.4) holds. Using this $\chi_{i}$ and (2.4), we have

$$
\begin{aligned}
\int_{0}^{t} \int_{R_{z}} w_{3} q d x d \eta= & \int_{0}^{t} \int_{R_{z}} \chi_{i}\left(w_{i, \eta}-\Delta w_{i}-g_{i} T\right) d x d \eta \\
\leq & \left(\int_{0}^{t} \int_{R_{z}} \chi_{i} \chi_{i} d x d \eta\right)^{1 / 2}\left(\int_{0}^{t} \int_{R_{z}} w_{i, \eta} w_{i, \eta} d x d \eta\right)^{1 / 2} \\
& +\left(\int_{0}^{t} \int_{R_{z}} \chi_{i, j} \chi_{i, j} d x d \eta\right)^{1 / 2}\left(\int_{0}^{t} \int_{R_{z}} w_{i, j} w_{i, j} d x d \eta\right)^{1 / 2} \\
& +g\left(\int_{0}^{t} \int_{R_{z}} \chi_{i} \chi_{i} d x d \eta\right)^{1 / 2}\left(\int_{0}^{t} \int_{R_{z}} T^{2} d x d \eta\right)^{1 / 2}
\end{aligned}
$$

By (2.1), we proceed to bound

$$
\begin{aligned}
\int_{0}^{t} \int_{R_{z}} w_{3} q d x d \eta \leq & \left(\frac{C}{\lambda} \int_{0}^{t} \int_{R_{z}} w_{3}^{2} d x d \eta\right)^{1 / 2}\left(\int_{0}^{t} \int_{R_{z}} w_{i, \eta} w_{i, \eta} d x d \eta\right)^{1 / 2} \\
& +\left(C \int_{0}^{t} \int_{R_{z}} w_{3}^{2} d x d \eta\right)^{1 / 2}\left(\int_{0}^{t} \int_{R_{z}} w_{i, j} w_{i, j} d x d \eta\right)^{1 / 2} \\
& +\frac{g}{\lambda}\left(C \int_{0}^{t} \int_{R_{z}} w_{3}^{2} d x d \eta\right)^{1 / 2}\left(\int_{0}^{t} \int_{R_{z}} T_{, i} T_{, i} d x d \eta\right)^{1 / 2} \\
\leq & A\left(-\frac{\partial E}{\partial z}\right)
\end{aligned}
$$

where $A=2 \sqrt{\frac{C}{\lambda}}\left(2+\frac{g}{\lambda}\right)$. 
For $\int_{0}^{t} \int_{R_{z}} w_{3, \eta} q d x d \eta$, with a derivation similar to (3.10), we obtain

$$
\begin{aligned}
\int_{0}^{t} \int_{R_{z}} w_{3, \eta} q d x d \eta \leq & \left(\frac{C}{\lambda} \int_{0}^{t} \int_{R_{z}} w_{3, \eta}^{2} d x d \eta\right)^{1 / 2}\left(\int_{0}^{t} \int_{R_{z}} w_{i, \eta} w_{i, \eta} d x d \eta\right)^{1 / 2} \\
& +\left(C \int_{0}^{t} \int_{R_{z}} w_{3, \eta}^{2} d x d \eta\right)^{1 / 2}\left(\int_{0}^{t} \int_{R_{z}} w_{i, j} w_{i, j} d x d \eta\right)^{1 / 2} \\
& +\frac{g}{\lambda}\left(C \int_{0}^{t} \int_{R_{z}} w_{3, \eta}^{2} d x d \eta\right)^{1 / 2}\left(\int_{0}^{t} \int_{R_{z}} T_{, i} T_{, i} d x d \eta\right)^{1 / 2} \\
\leq & B\left(-\frac{\partial E}{\partial z}\right)
\end{aligned}
$$

where $B=2 \sqrt{C}\left(2 \sqrt{\lambda}+\frac{g}{\lambda^{3 / 2}}\right)$. On applying Schwarz's inequality, the arithmetic-geometric mean inequality, (2.1), and (2.4) on the other remaining terms on the right in (3.7), it then follows that

$$
E(z, t) \leq K\left(-\frac{\partial E}{\partial z}\right)
$$

where $K$ is a computable constant. Upon integration we have

$$
E(z, t) \leq E(0, t) e^{-z / K}
$$

In order to make the exponential decay inequality (3.13) explicit, we require a bound for $E(0, t)$ in terms of the boundary data. In the next section we will indicate a procedure for obtaining bounds for the total weighed energy $E(0, t)$.

\section{Bounds for $E(0, t)$}

In this section we sketch how one can derive the total energy needed to complete our decay results.

We first note from (3.12) that

$$
E(0, t) \leq-K \frac{\partial E}{\partial z}(0, t)
$$

Thus, we must bound $-\frac{\partial E}{\partial z}(0, t)$, which implies that we need to bound

$$
\int_{0}^{t} \int_{R} T_{, i} T_{, i} d x d \eta, \int_{0}^{t} \int_{R} w_{i, \eta} w_{i, \eta} d x d \eta, \int_{0}^{t} \int_{R} w_{i, j} w_{i, j} d x d \eta
$$

We first derive a bound for $\int_{0}^{t} \int_{R} T_{, i} T_{, i} d x d \eta$ in terms of $\int_{0}^{t} \int_{R} w_{i, j} w_{i, j} d x d \eta$, then seek a bound for $\int_{0}^{t} \int_{R} w_{i, j} w_{i, j} d x d \eta$ in terms of data, and finally bound $\int_{0}^{t} \int_{R} w_{i, \eta} w_{i, \eta} d x d \eta$ in terms of data. We will use arguments similar to those 
employed in $[11,13,12]$ to bound $\int_{0}^{t} \int_{R} T_{, i} T_{, i} d x d \eta$. Thus

$$
\begin{aligned}
& \int_{0}^{t} \int_{R} T_{, i} T_{, i} d x d \eta \\
= & -\int_{0}^{t} \int_{D} F T_{, 3} d A d \eta-\int_{0}^{t} \int_{R} T\left(T_{, \eta}+u_{i} T_{, i}\right) d x d \eta \\
= & -\int_{0}^{t} \int_{D} F T_{, 3} d A d \eta+\frac{1}{2} \int_{0}^{t} \int_{D} f_{3} F^{2} d A d \eta-\left.\frac{1}{2} \int_{R} T^{2} d x\right|_{\eta=t} .
\end{aligned}
$$

To bound the first term in (4.2) we set

$$
S=F\left(x_{1}, x_{2}, t\right) e^{-\gamma z}
$$

for some positive $\gamma$. Then

$$
\begin{aligned}
& -\int_{0}^{t} \int_{D} F T_{, 3} d A d \eta \\
= & \int_{0}^{t} \oint_{\partial R} S T_{, i} n_{i} d s d \eta \\
= & \int_{0}^{t} \int_{R} S_{, i} T_{, i} d x d \eta+\int_{0}^{t} \int_{R} S\left(T_{, \eta}+u_{i} T_{, i}\right) d x d \eta \\
= & \int_{0}^{t} \int_{R} S_{, i} T_{, i} d x d \eta+\left.\int_{R} S T d x\right|_{\eta=t}-\int_{0}^{t} \int_{R} T S_{, \eta} d x d \eta \\
& -\int_{0}^{t} \int_{R} S_{, i}\left(w_{i}+v_{i}\right) T d x d \eta,
\end{aligned}
$$

where $d s$ is the element of surface area on $\partial R$. Inserting (4.4) into (4.2) and using the arithmetic-geometric mean inequality and the inequality (2.1) we have for some positive $\hat{\epsilon}_{1}, \hat{\epsilon}_{2}$, and $\hat{\epsilon}_{3}$

$$
\begin{aligned}
& {\left[1-\left(\frac{\hat{\epsilon}_{1}}{2}+\frac{\hat{\epsilon}_{2}}{2}+\frac{\hat{\epsilon}_{3}}{2}\right)\right] \int_{0}^{t} \int_{R} T_{, i} T_{, i} d x d \eta } \\
\leq & \left(\frac{1}{2 \hat{\epsilon}_{1}}+\frac{|V|_{M}^{2}}{2 \lambda \hat{\epsilon}_{3}}\right) \int_{0}^{t} \int_{R} S_{, i} S_{, i} d x d \eta+\frac{1}{2 \lambda \hat{\epsilon}_{2}} \int_{0}^{t} \int_{R} S_{, \eta}^{2} d x d \eta \\
& +\left.\frac{1}{2} \int_{R} S^{2} d x\right|_{\eta=t}+T_{M}\left(\int_{0}^{t} \int_{R} S_{, i} S_{, i} d x d \eta \int_{0}^{t} \int_{R} w_{i} w_{i} d x d \eta\right)^{1 / 2}+\text { data. }
\end{aligned}
$$

Choosing $\hat{\epsilon}_{1}=1 / 2, \hat{\epsilon}_{2}=1 / 4, \hat{\epsilon}_{3}=1 / 4$, by (2.1) and the arithmetic-geometric mean inequality we can write for some positive $\epsilon$

$$
\int_{0}^{t} \int_{R} T_{, i} T_{, i} d x d \eta \leq \epsilon \int_{0}^{t} \int_{R} w_{i, j} w_{i, j} d x d \eta+\text { data }
$$

where the data involve parameters $\lambda, T_{M},|V|_{M}$ and $\int_{0}^{t} \int_{R} S_{, i} S_{, i} d x d \eta$, $\int_{0}^{t} \int_{R} S_{, \eta}^{2} d x d \eta,\left.\int_{R} S^{2} d x\right|_{\eta=t}$ and $\int_{0}^{t} \int_{D} f_{3} F^{2} d A d \eta$ which are clearly data. 
To obtain a bound for $\int_{0}^{t} \int_{R} w_{i, j} w_{i, j} d x d \eta$, by the triangle inequality we have

$$
\begin{aligned}
\int_{0}^{t} \int_{R} w_{i, j} w_{i, j} d x d \eta \leq & 2 \int_{0}^{t} \int_{R}\left(w_{i}-\tilde{w}_{i}\right)_{, j}\left(w_{i}-\tilde{w}_{i}\right)_{, j} d x d \eta \\
& +2 \int_{0}^{t} \int_{R} \tilde{w}_{i, j} \tilde{w}_{i, j} d x d \eta
\end{aligned}
$$

where

$$
\tilde{w}_{i}=\left[f_{i}\left(x_{1}, x_{2}, t\right)-V \delta_{i 3}\right] e^{-\sigma z}
$$

for some positive $\sigma$. Using integration by parts and the lateral surface boundary condition (1.5), we obtain

$$
\begin{aligned}
& \int_{0}^{t} \int_{R}\left(w_{i}-\tilde{w}_{i}\right)_{, j}\left(w_{i}-\tilde{w}_{i}\right)_{, j} d x d \eta \\
(4.9)= & -\int_{0}^{t} \int_{R}\left(w_{i}-\tilde{w}_{i}\right) \Delta\left(w_{i}-\tilde{w}_{i}\right) d x d \eta \\
= & -\int_{0}^{t} \int_{R}\left(w_{i}-\tilde{w}_{i}\right)\left[\left(w_{i, \eta}-\tilde{w}_{i, \eta}\right)+q_{, i}-g_{i} T+\left(\tilde{w}_{i, \eta}-\Delta \tilde{w}_{i}\right)\right] d x d \eta .
\end{aligned}
$$

Upon integration by parts and application of Schwarz's inequality, the arithmetic-geometric mean inequality and (2.1), we find for some positive $\gamma_{1}$ and $\gamma_{2}$,

$$
\begin{aligned}
& \left(1-\frac{\gamma_{1}+\gamma_{2}}{2 \lambda}\right) \int_{0}^{t} \int_{R}\left(w_{i}-\tilde{w}_{i}\right)_{, j}\left(w_{i}-\tilde{w}_{i}\right)_{, j} d x d \eta \\
\leq & \frac{g}{2 \gamma_{1} \lambda} \int_{0}^{t} \int_{R} T_{, i} T_{, i} d x d \eta+\frac{1}{2 \gamma_{2}} \int_{0}^{t} \int_{R}\left(\tilde{w}_{i, \eta}-\Delta \tilde{w}_{i}\right)\left(\tilde{w}_{i, \eta}-\Delta \tilde{w}_{i}\right) d x d \eta+\text { data. }
\end{aligned}
$$

Substituting (4.7) into (4.6) and inserting the result back into (4.10), we conclude that

$$
\left(1-\frac{\gamma_{1}+\gamma_{2}}{2 \lambda}-\frac{g \epsilon}{\gamma_{1} \lambda}\right) \int_{0}^{t} \int_{R}\left(w_{i}-\tilde{w}_{i}\right)_{, j}\left(w_{i}-\tilde{w}_{i}\right)_{, j} d x d \eta \leq \text { data. }
$$

Choosing $\gamma_{1}=\lambda / 4, \gamma_{2}=\lambda / 4, \epsilon=\gamma_{1} \lambda /(4 g)$ and combining (4.7), we have

$$
\int_{0}^{t} \int_{R} w_{i, j} w_{i, j} d x d \eta \leq \text { data. }
$$

It then follows from (4.6) that we find

$$
\int_{0}^{t} \int_{R} T_{, i} T_{, i} d x d \eta \leq \text { data. }
$$


Turning now to the bound for $\int_{0}^{t} \int_{R} w_{i, \eta} w_{i, \eta} d x d \eta$, by the triangle inequality we have

$$
\begin{aligned}
\left(\int_{0}^{t} \int_{R} w_{i, \eta} w_{i, \eta} d x d \eta\right)^{1 / 2} \leq & \left(\int_{0}^{t} \int_{R}\left(w_{i}-\tilde{w}_{i}\right)_{, \eta}\left(w_{i}-\tilde{w}_{i}\right)_{, \eta} d x d \eta\right)^{1 / 2} \\
& +\left(\int_{0}^{t} \int_{R} \tilde{w}_{i, \eta} \tilde{w}_{i, \eta} d x d \eta\right)^{1 / 2}
\end{aligned}
$$

We first note that

$$
\begin{aligned}
& \int_{0}^{t} \int_{R}\left(w_{i}-\tilde{w}_{i}\right)_{, \eta}\left(w_{i}-\tilde{w}_{i}\right)_{, \eta} d x d \eta \\
= & \int_{0}^{t} \int_{R}\left(w_{i}-\tilde{w}_{i}\right)_{, \eta}\left(-q_{, i}+\Delta\left(w_{i}-\tilde{w}_{i}+\tilde{w}_{i}\right)+g_{i} T-\tilde{w}_{i, \eta}\right) d x d \eta .
\end{aligned}
$$

An application of Schwarz's inequality and the arithmetic-geometric mean inequality gives for some positive $\delta_{1}, \delta_{2}$ and $\delta_{3}$

$$
\begin{aligned}
& \left(1-\frac{\delta_{1}+\delta_{2}+\delta_{3}}{2}\right) \int_{0}^{t} \int_{R}\left(w_{i}-\tilde{w}_{i}\right)_{, \eta}\left(w_{i}-\tilde{w}_{i}\right)_{, \eta} d x d \eta \\
\leq & \frac{1}{2 \delta_{1}} \int_{0}^{t} \int_{R} \Delta \tilde{w}_{i} \Delta \tilde{w}_{i} d x d \eta+\frac{g^{2}}{2 \delta_{2}} \int_{0}^{t} \int_{R} T^{2} d x d \eta \\
& +\frac{1}{2 \delta_{3}} \int_{0}^{t} \int_{R} \tilde{w}_{i, \eta} \tilde{w}_{i, \eta} d x d \eta,
\end{aligned}
$$

where we have dropped a negative spatial integral term. For instance, taking $\delta_{1}=1 / 4, \delta_{2}=1 / 2, \delta_{3}=1 / 4$ and using (2.1), we find

$$
\int_{0}^{t} \int_{R}\left(w_{i}-\tilde{w}_{i}\right)_{, \eta}\left(w_{i}-\tilde{w}_{i}\right)_{, \eta} d x d \eta \leq \frac{2 g^{2}}{\lambda} \int_{0}^{t} \int_{R} T_{, i} T_{, i} d x d \eta+\text { data. }
$$

Combining (4.6), (4.14), and (4.17), we have

$$
\int_{0}^{t} \int_{R} w_{i, \eta} w_{i, \eta} d x d \eta \leq \text { data. }
$$

When these results (4.12), (4.13), and (4.18) are inserted into (4.1), the bound for $E(0, t)$ in terms of data is obtained.

Acknowledgment. The author thanks a referee for constructive comments and suggestions on improving an earlier version of manuscript.

\section{References}

[1] K. A. Ames, L. E. Payne, and P. W. Schaefer, Spatial decay estimates in time-dependent Stokes flow, SIAM J. Math. Anal. 24 (1993), no. 6, 1395-1413.

[2] I. Babuǒska and A. K. Aziz, Survey lectures on the mathematical foundations of the finite element method, The mathematical foundations of the finite element method with applications to partial differential equations (Proc. Sympos., Univ. Maryland, Baltimore, Md., 1972), pp. 1-359. Academic Press, New York, 1972. 
[3] C. O. Horgan, Recent developments concerning Saint-Venant's principle: an update, Appl. Mech. Rev. 42 (1989), no. 11, 295-303.

[4] — Recent developments concerning Saint-Venant's principle: a second update, Appl. Mech. Rev. 49 (1996), 101-111.

[5] C. O. Horgan and J. K. Knowles, Recent developments concerning Saint-Venant's principle, Adv. in Appl. Mech. 23 (1983), 179-69.

[6] C. O. Horgan and L. T. Wheeler, Spatial decay estimates for the Navier-Stokes equations with application to the problem of entry flow, SIAM J. Appl. Math. 35 (1978), no. 1, 97-116.

[7] O. A. Ladyzhenskaya and V. A. Solonnikov, Some problems of vector analysis and generalized formulations of boundary-value problems for the Navier-Stokes equations, Russ. Math. Surveys 28 (1973), 43-82.

[8] C. Lin and L. E. Payne, Spatial decay bounds in time-dependent pipe flow of an incompressible viscous fluid, SIAM J. Appl. Math. 65 (2004), no. 2, 458-474.

[9] L. E. Payne, Isoperimetric inequalities and their applications, SIAM Rev. 9 (1967), 453-488.

[10] A bound for the optimal constant in an inequality of Ladyzhenskaya and Solonnikov, IMA J. Appl. Math. 72 (2007), no. 5, 563-569.

[11] L. E. Payne and J. C. Song, Spatial decay for a model of double diffusive convection in Darcy and Brinkman flows, Z. Angew. Math. Phys. 51 (2000), no. 6, 867-880.

[12] S S S Satial decay in a double diffusive convection problem in Darcy flow, J. Math. Anal. Appl. 330 (2007), no. 2, 864-875.

[13] Spatial decay bounds for double diffusive convection in Brinkman flow, J. Differential Equations 244 (2008), no. 2, 413-430.

[14] J. C. Song, Improved decay estimates in time-dependent Stokes flow, J. Math. Anal. Appl. 288 (2003), no. 2, 505-517.

[15] B. Straughan, Stability and Wave Motion in Porous Media, Springer, New York, 2008.

Department of Applied Mathematics

HANYANG UNIVERSITY

ANSAN 426-791, KoreA

E-mail address: jcsong@hanyang.ac.kr 